\title{
Ultrastructural and immunocytochemical study of a case of invasive cribriform breast carcinoma
}

\author{
C A WELLS, D J P FERGUSON \\ From the Nuffield Department of Pathology, University of Oxford, John Radcliffe Hospital, Oxford
}

SUMMARY A case of recurrent invasive cribriform carcinoma of the breast mimicked the histological and mucin staining characteristics of adenoid cystic carcinoma. The diagnosis was based on negative immunocytochemical staining for laminin and ultrastructural evidence of luminal differentiation by cells lining the cystic spaces. Accurate characterisation of this type of breast tumour can be facilitated by retrospective immunocytochemical or ultrastructural examination, or both.

One area in which the characterisation of invasive breast tumours can be difficult is the differentiation between invasive cribriform' ${ }^{1}$ and adenoid cystic $^{2}$ carcinoma of the breast. Although these tumours have similar histological appearances, it was thought that they could be differentiated by their characteristic mucin staining and the absence of axillary node metastases. Both of these criteria, however, have been questioned. ${ }^{34}$

We report a case of recurrent carcinoma of the breast which had the histological and mucin staining characteristics of adenoid cystic carcinoma that was shown to be invasive cribriform carcinoma by immunocytochemistry and electron microscopy.

\section{Case report}

A 39 year old woman presented in 1975 with a breast lump and bleeding from the nipple. A biopsy was performed and cribriform intraduct carcinoma with early invasion was diagnosed. A simple mastectomy was performed. In 1977 and 1983 nodules were removed from beneath the mastectomy scar. These recurrences were considered to be adenoid cystic carcinoma. In 1986 the patient presented with a further two nodules beneath the mastectomy scar. These were removed. The patient remained well and free from recurrent or metastatic disease at the time of writing.

\section{Material and methods}

The specimens from the last operation were fixed in

Accepted for publication 8 July 1987 neutral buffered formalin and blocks were processed for histological examination. Sections were cut and stained with haematoxylin and eosin, alcian blue combined with periodic acid Schiff (PAS) after diastase for mucins, and by the indirect immunoperoxidase method using a polyclonal antibody directed against laminin (Bethseda Research Laboratories, Cambridge), and monoclonal antibodies to various cytokeratins. Small cubes were retrieved from the formalin fixed tissue and processed for electron microscopy by standard techniques. Thin sections were stained with uranyl acetate and lead citrate before being examined in a JEOL $100 \mathrm{CX}$ electron microscope.

\section{Results}

Both nodules showed a pattern of cribriform islands of tumour cells containing cyst-like spaces (fig 1) and were surrounded by a compressed pseudo capsule of connective tissue with small areas of infiltration. Mucin staining identified two populations of cystic spaces, one of which stained for acid mucopolysaccharides (alcian blue positive) and the other for neutral mucopolysaccharides (PAS positive) (fig 1 , insert).

The cystic spaces were uniformly negative with the laminin immunostaining, although there were areas of positivity around blood vessels (fig 2a). Staining for the epithelial cytokeratins KL-1 and CAM 5.2 showed strong positivity within the tumour cells (fig $2 \mathrm{~b}$ ).

Electron microscopy showed that the tumour consisted of islands of relatively uniform cells subtending cyst-like spaces of various sizes (fig 3 ). These spaces were lined by cells showing luminal differentiation with junctional complexes consisting of tight and intermediate junctions plus desmosomes (fig 3 and 
Fig 1 Survey view of a typical area of invasive cribriform carcinoma. (Haematoxylin and eosin.) Insert: mucin staining showing two different types of mucin in adjacent lumina. (Arrows indicate PAS positive lumina) (Alcian blue/PAS.)

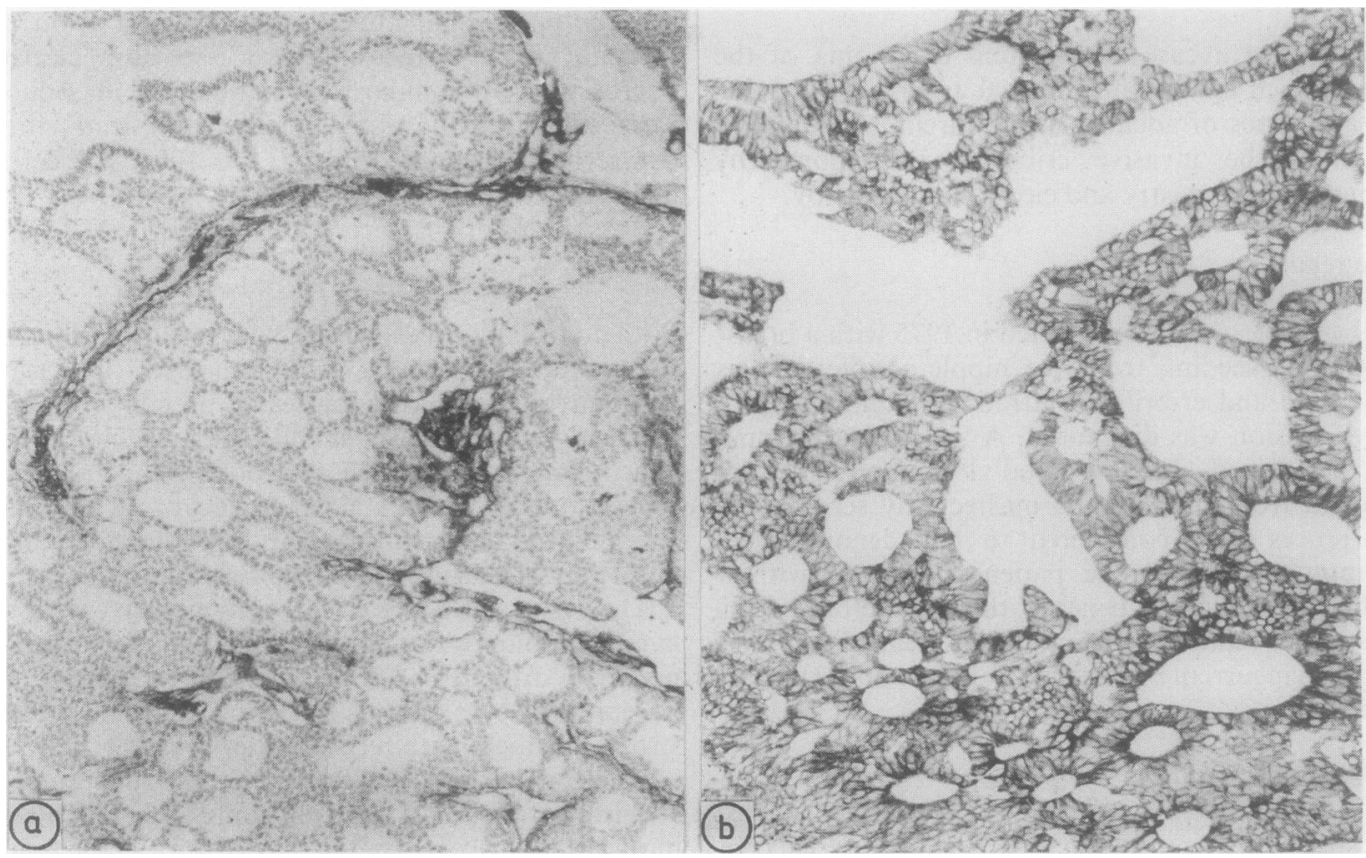

Fig 2 (a) Laminin staining showing negative cystic spaces. Reaction product is associated with blood vessel basement membranes. (Laminin immunoperoxidase.)

(b) Cytokeratin staining showing all cells staining as epithelial cells (CAM 5.2 immunoperoxidase.) 


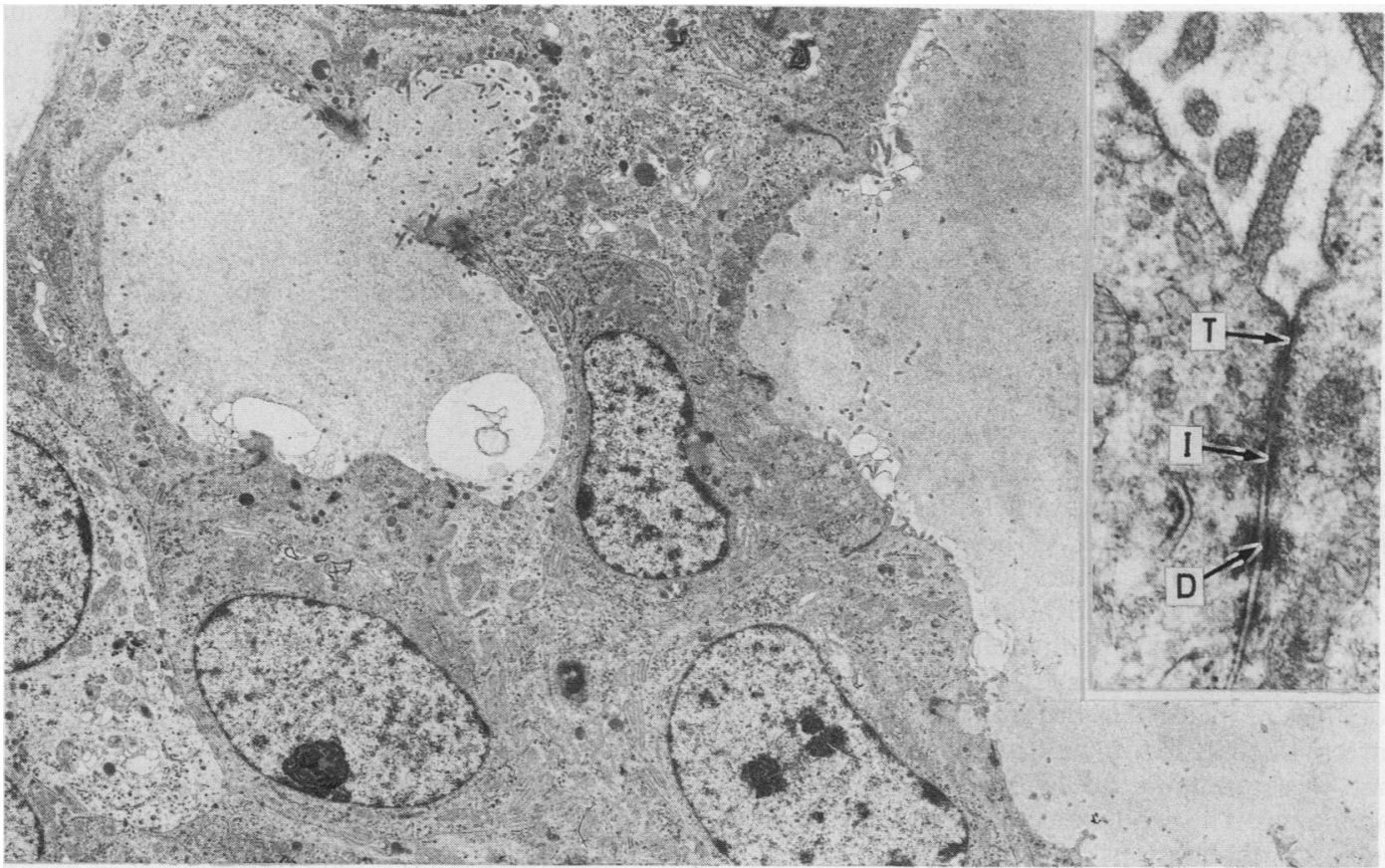

Fig 3 Low power electron micrograph showing several cystic spaces subtended by tumour cells. Insert: detail from periphery of cystic space showing luminal differentiation characterised by microvilli and a junctional complex. $T=$ tight junction; $I=$ intermediate junction; $D=$ desmosome.

insert). In addition, several cells possessed large numbers of mucin containing vacuoles of variable electron density, concentrated in the apical cytoplasm adjacent to the cystic spaces.

At the periphery of the tumour, the cells were in direct contact with the connective tissue or separated from it by mucin (fig 4). There was no evidence of an intact basal lamina, although a few remnants were observed in certain areas.

\section{Discussion}

The tissue examined in this report represents the third local recurrence of a breast tumour with histological features similar to those of adenoid cystic carcinoma: this diagnosis had been made on three separate occasions by experienced histopathologists. When this latest recurrence was examined by immunocytochemistry and electron microscopy, however, the appearances were found to be those of invasive cribriform carcinoma. The present case mimics adenoid cystic carcinoma in exhibiting both alcian blue and PAS positive lumina and cannot be differentiated by different mucin staining patterns. In this respect it is similar to a case of in situ cribriform carcinoma described by Harris. ${ }^{3}$ The good prognosis described for invasive cribriform carcinoma ${ }^{1}$ is exemplified in the present case where, although there have been three

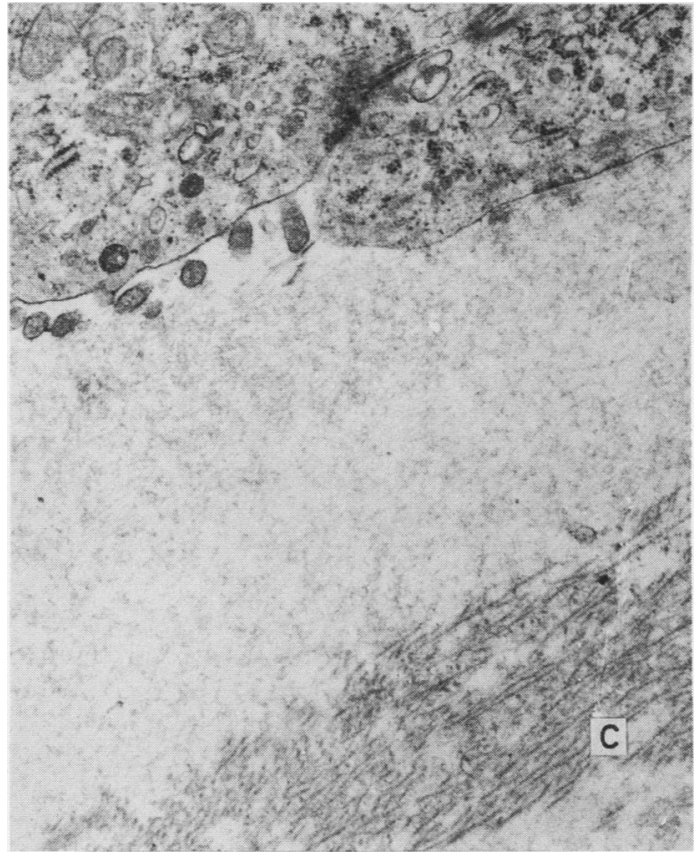

Fig 4 Micrograph from periphery of lesion showing tumour cells adjacent to connective tissue with no intervening basal lamina. $C=$ collagen. 
instances of local recurrence, the woman was well with no evidence of systemic disease 12 years after the initial diagnosis.

To the best of our knowledge this is the first description of the ultrastructural features of invasive cribriform carcinoma of the breast since its description by Page et al. ${ }^{1}$ It appears to be a well differentiated tumour with the formation of lumina characterised by junctional complexes and microvilli around all the cystic spaces. The formation of lumina is similar to that reported for both tubular ${ }^{5}$ and mucoid ${ }^{6}$ carcinoma of the breast. Interestingly, the ultrastructural review of breast tumours by Fisher ${ }^{5}$ showed that the category of adenoid cystic carcinoma contained two tumours with ultrastructural features similar to those of the case reported here. As these tumours lacked the characteristic ultrastructural features (such as basal lamina formation) of adenoid cystic carcinoma the term "adenoid cystic configuration" was proposed. With our present knowledge these cases were probably examples of invasive cribriform carcinoma that were unrecognised at that time. It was proposed by Fisher that these tumours could be variants of tubular carcinoma. ${ }^{5}$ This would be consistent with the ultrastructural observations of lumen formation in our study.

The absence of a basal lamina around the islands of tumour cells and inside the cyst-like spaces is consistent with the negative laminin staining, and contrasts with the strong positive staining observed in adenoid cystic carcinoma of the breast and other organs. ${ }^{47}$ This also differentiates the tumour from in situ cribriform carcinoma, which possesses an intact basal lamina. ${ }^{3}$

In those tumours with a "cribriform pattern" or an "adenoid cystic configuration", where accurate diagnosis is difficult, immunocytochemical staining for basement membrane materials or ultrastructural examination is recommended.

The authors wish to thank Mr A Heryet and Mrs Y Smart for technical assistance. KL-I was kindly supplied by J Brochier, Lyon, France and CAM 5.2 by C Martin, Imperial Cancer Research Fund, London.

\section{References}

1 Page DL, Dixon JM, Anderson TJ, Lee D, Stewart HJ. Invasive cribriform carcinoma of the breast. Histopathology 1983;7:52536.

2 Anthony PP, James PD. Adenoid cystic carcinoma of the breast: prevalence, diagnostic criteria, and histogenesis. J Clin Pathol 1975;28:647-55.

3 Harris M. Pseudoadenoid cystic carcinoma of the breast. Arch Pathol Lab Med 1977;101:307-9.

4 Wells CA, Nicol S, Ferguson DJP. Adenoid cystic carcinoma of the breast: a case with axillary lymph node metastasis. Histopathology 1986;10:415-24.

5 Fisher B. Ultrastructure of human breast and its disorders. $\mathrm{Am} \mathrm{J}$ Clin Pathol 1976;66:291-374.

6 Ferguson DJP, Anderson TJ, Wells CA, Battersby S. An ultrastructural study of mucoid carcinoma of the breast: variability of cytoplasmic features. Histopathology 1986;10:1219-30.

7 D'Ardenne AJ, Kirkpatrick P, Wells CA, Davies JD. Laminin and fibronectin in adenoid cystic carcinomas. J Clin Pathol 1985;39:138-44.

Requests for reprints to: Dr C A Wells, Nuffield Department of Pathology, John Radcliffe Hospital, Level 1, Headington, Oxford OX3, 9DU, England. 